\title{
Experiencia del proceso de intervención familiar de los actores participantes en la Escuela para Familias del ICBF- Jamundí, Colombia
}

\author{
Katherin Viviana Silva-Minotta \\ Trabajadora social \\ Save the Children. Cali, Colombia \\ https://orcid.org/0000-0002-3996-4216• katherin.silva@savethechildren.org \\ María José Forero-Izquierdo \\ Magister Educación y Desarrollo Humano. Trabajadora Social \\ Alcaldía Municipal de Jamundí. Jamundí, Colombia \\ https://orcid.org/0000-0003-2346-4660 • mforeroizquierdo@gmail.com \\ Jimena del Pilar Jaramillo-Jaramillo \\ Magister en Gerontología. Trabajadora Social \\ Universidad de Deusto. Bilbao, España \\ https://orcid.org/0000-0003-0010-2616•jimenajaramillo@opendeusto.es
}

\section{Resumen}

Este artículo contiene los resultados del proceso de sistematización de la Escuela para Familias, realizado en el Instituto Colombiano de Bienestar Familiar en el Centro Zonal Jamundí, durante los años 2018-2019. Esta sistematización se elaboró con el propósito de recuperar, interpretar y aportar al proceso, concediendo voz a los sujetos que participaron en la experiencia (familias, practicante y profesionales). La recolección de información y el análisis se construyó a partir de entrevistas y microrrelatos, que posteriormente, se interpretaron y repensaron a la luz de la teoría.

La sistematización arrojó algunos resultados como la importancia de la articulación institucional e interdisciplinaria con base en los procesos de intervención familiar, la especificidad de trabajo social, y la envergadura en la recuperación de las experiencias desde los sujetos para generar un conocimiento y aprendizaje significativo. El acercamiento inicial permitió conocer los elementos fundamentales de la experiencia, y el proceso se remontó a la práctica pre profesional en el nivel comunitario, vivenciada por las estudiantes de trabajo social de la Institución Universitaria Antonio José Camacho en el centro de práctica.

Palabras clave: Sistematización de experiencias; Desarrollo familiar; Intervención familiar; Trabajo Social.

Recibido: 25/03/2021| Aprobado: 09/11/2021| Publicado: 01/01/2022

(i) (2) Esta obra está bajo una Licencia Creative Commons Atribución-NoComercial-CompartirIgual 4.0 Internacional.

Financiación o proveniencia del artículo: Artículo derivado del Proyecto de sistematización de la Escuela para Familias, realizado en el Instituto Colombiano de Bienestar Familiar en el período 2018-2019, sede Jamundí.

¿Cómo citar este artículo? / How to quote this article?

Silva-Minotta, K. V, Forero-Izquierdo, M. J., y Jaramillo-Jaramillo, J. P. (2022). Experiencia del proceso de intervención familiar de los actores participantes en la Escuela para Familias del ICBF-Jamundí, Colombia. Prospectiva. Revista de Trabajo Social e intervención social, (33), 305-328. doi: 10.25100/prts.v0i33.11097.

e-ISSN: 2389-993X • https:/ / doi.org/10.25100/prts.v0i33.11097• Universidad del Valle - Cali, Colombia 
Silva-Minotta, Forero-Izquierdo y Jaramillo-Jaramillo

\title{
Experience of the Family Intervention Process of the Participating Actors in the School for Families of the ICBF- Jamundí, Colombia
}

\begin{abstract}
This article contains the results of the systematization process of the School for Families, carried out at the Colombian Institute of Family Welfare in the Centro Zonal Jamundí, during the years 2018-2019. This systematization was developed with the purpose of recovering, interpreting and contributing to the process, giving a voice to the subjects who participated in the experience (families, practitioner and professionals). The collection of information and the analysis was built from interviews and micro-stories, which were subsequently interpreted in light of the theory.

The systematization yielded some results such as the importance of institutional and interdisciplinary articulation based on family intervention processes, the specificity of social work, and the scope in the recovery of experiences from the subjects to generate meaningful knowledge and learning. The initial approach offered knowledge about the fundamental elements of the experience, and the process went back to the pre-professional practice at the community level, experienced by the social work students of the Antonio Jose Camacho University Institution in the practice center.
\end{abstract}

Keywords: Systematization of experiences; Family development; Family intervention, Social work.

Sumario: 1. Introducción, 2. Contexto de la Experiencia, 3. Objeto y ejes de la Sistematización, 4. Metodología, 5. Hallazgos, 6. Conclusiones, 7. Referencias bibliográficas. 


\section{Introducción}

El presente documento expone los resultados de la sistematización de experiencias en el proceso de intervención familiar educativa Escuela para Familias del Instituto Colombiano de Bienestar Familiar (ICBF) desarrollado durante los años 2018- 2019 en el Centro Zonal Jamundí, Valle del Cauca. Se consignan las reflexiones desde las narrativas de los sujetos que participaron en la construcción de la experiencia (familias, practicante y profesionales), reconociendo elementos que incorporan los procesos de intervención familiar a través de la construcción de perspectivas compartidas sobre la formulación, la implementación y los resultados posteriores a la experiencia, teniendo en cuenta el intercambio de los aprendizajes con la intención de resaltar elementos del proceso que aportaron en la transformación de los sujetos y su entorno desde sus dinámicas y percepciones.

Se pretende visibilizar a las familias como sujetos sociales que, desde sus dinámicas propias y su diversidad, pueden ser protagonistas de su propio cambio a partir del fortalecimiento de sus prácticas. De igual manera, facilitar propuestas metodológicas para la elaboración de un modelo de intervención familiar como herramienta que contribuya a la construcción de saberes de la profesión de Trabajo Social con relación al desarrollo familiar desde experiencias particulares, pues los estudios sobre familia apoyan las formas del funcionamiento familiar, el reconocimiento del cambio y la diversidad.

A su vez, los procesos de sistematización son fundamentales en instituciones como el Instituto Colombiano de Bienestar Familiar (ICBF), porque orientan la intervención a la construcción de reflexiones sobre sus experiencias. En la Escuela para Familias de los centros zonales, específicamente en el Centro Zonal Jamundí, la intención de la intervención fue aportar al reconocimiento de los elementos incorporados en la construcción, el desarrollo y las transformaciones que generaron los sujetos desde sus prácticas. Por otro lado, el facilitar propuestas metodológicas para la elaboración de un modelo de intervención familiar que contribuya a la inclusión de temas no abordados y contemplados dentro del marco institucional (lineamientos del ICBF).

Por consiguiente, el intercambio de los aprendizajes en la Escuela para Familias aportó a otros Centros Zonales en la reproducción de elementos metodológicos. Asimismo, en lograr la autorreflexión que hacen los sujetos que impulsan una experiencia de acción social o educativa, a partir del reconocimiento de los saberes (Torres-Carrillo, 2010, p. 11).

Ciertamente, la Escuela para Familias aborda desde una postura transdisciplinar e integradora, las problemáticas de las familias, que son comprendidas como sujetos portadores de cultura de los distintos contextos sociales de influencia. Además, promueve la diversidad de las familias con la atención diferencial y las acciones mediadoras de los 
funcionarios y practicantes, que les permite visibilizarse para exigir la garantía de sus derechos, en el Estado Social de Derecho y favorecer las relaciones democráticas.

De esta manera, la sistematización posibilita destacar los procesos que se están desarrollando en el Instituto Colombiano de Bienestar Familiar (ICBF) con la Escuela para Familias del Centro Zonal Jamundí, en el que se reconoce a las familias como sujetos sociales protagonistas de su transformación desde sus dinámicas y fortalecimiento de sus prácticas. Adicionalmente, para la profesión de Trabajo Social, la reconstrucción de la experiencia se originó con las prácticas de Trabajo Social en el Instituto Colombiano de Bienestar Familiar (ICBF), Centro Zonal Jamundí, y en otros centros zonales, y estuvo orientada al acompañamiento, apoyo e intervención familiar a partir de la Escuela para Familias. Allí se exploraron lecciones aprendidas del proceso de formación familiar educativo y la intervención que genera transformaciones en los sujetos y sus entornos de acción.

El proceso de sistematización permite la expansión de aprendizajes adquiridos para el desarrollo de nuevos saberes desde el Trabajo Social, con relación al desarrollo y fortalecimiento familiar desde experiencias particulares, pues los estudios actuales sobre familia legitiman nuevas formas de estructura y funcionamiento familiar, distinguen los cambios y la diversidad social.

Se destaca que, la propuesta de sistematización surgió de la práctica pre profesional como estudiantes de Trabajo Social de la Institución Universitaria Antonio José Camacho, se desarrolló en el Instituto Colombiano de Bienestar Familiar (ICBF) en el Centro Zonal (CZ) Jamundí de la ciudad de Cali en el Valle del Cauca, en el segundo período del año 2018 y el primero del año 2019. De acuerdo con Ghiso (1998) toda sistematización está antecedida por una práctica, y visibiliza al sujeto como ser que percibe la realidad y produce conocimiento.

Durante el proceso de prácticas, se realizó el acompañamiento en el apoyo y la orientación familiar de la Escuela para Familias, participando en el diseño y la aplicación de talleres y temáticas pertinentes que tuvieron la intención de proporcionar las herramientas necesarias para el desarrollo familiar.

En ese orden de ideas, el acompañamiento en el apoyo y la orientación familiar de la Escuela para Familias que realizan los Centros Zonales del Instituto Colombiano de Bienestar Familiar (ICBF) se ha ejecutado desde el proceso misional de protección de los niños, niñas y adolescentes (NNA) como medida correctiva establecida por las defensorías para sus familias en atención de los derechos inobservados, amenazados o vulnerados.

Ahora bien, en los registros identificados se contempló que desde el 2016 se han desarrollado cada año dos ciclos de Escuela para Familias en el Centro Zonal Jamundí: el 
Silva-Minotta, Forero-Izquierdo y Jaramillo-Jaramillo

ciclo I iniciando el año y el ciclo II finalizándolo. En cada ciclo se efectuaron seis encuentros de dos horas cada uno, durante dos meses. Para el año 2018, se realizaron un total de dos ciclos de trece encuentros a los que asistieron treinta y dos personas; en el 2019 se efectuaron tres ciclos, con un total de veintidós encuentros, asistieron setenta y ocho personas. De manera general, se realizaron talleres y dinámicas de integración en las que se abordaron temáticas como: pautas de crianza, prevención del suicidio, identidad familiar y principios y valores.

Justamente en el año 2019, se desarrollaron talleres participativos y dinámicos en los que se abordaron los siguientes temas: corresponsabilidad familiar, comunicación asertiva, pautas de crianza, prevención del suicidio y rutas de atención. Dicho lo anterior, es importante enunciar que, las familias durante las sesiones expresaron la necesidad de abordar otros temas como la violencia intrafamiliar, el consumo de sustancias psicoactivas entre otros, también se evaluaron las actividades mediante un formato en el que se consultó a los asistentes su percepción y sugerencias para los próximos talleres. Por lo tanto, las evaluaciones fueron un insumo que posibilitó la generación de cambios en las estrategias y las metodologías de los talleres.

Cabe mencionar que la Escuela para Familias no tiene un período de tiempo determinado, pues depende de las temáticas abordadas por el o la profesional que conforme el equipo psicosocial (psicólogo(a), Trabajador(a) Social y/o practicantes) responsable de esta, el cual es asignado por el coordinador o la coordinadora del Centro Zonal, para el diseño y la ejecución de los talleres.

A propósito, en el proceso surgieron las siguientes inquietudes: ¿Por qué, si existen unas líneas de acción para el trabajo con familias en el ICBF según grupos etarios del 2010, no se implementa en su totalidad en la Escuela para Familias del Centro Zonal Jamundí?, ¿Cómo lograr que las familias asistan y participen en las actividades de la Escuela para Familias, sin que haya de por medio una sanción?, ¿Cómo lograr el empoderamiento de las familias en el proceso de construcción de las actividades de la Escuela para familias?, ¿Qué de esta experiencia es significativo y debe ser rescatado por los sujetos sociales que participan en la experiencia?, de las familias que participaron ¿Cuáles fueron los procesos de difusión de la experiencia en sus comunidades?

\section{Contexto de la experiencia}

El Centro Zonal Jamundí se encuentra ubicado en la Carrera 13 \# 10 - 21 del barrio la Estación en el municipio de Jamundí, uno de los 42 municipios del departamento del Valle del Cauca. El territorio está comprendido mayormente por zonas rurales, por lo que, en el proceso, las familias desisten de la continuidad por la dificultad del desplazamiento al centro zonal, al no disponer de recursos económicos. En el transcurso del proceso se registró 
Silva-Minotta, Forero-Izquierdo y Jaramillo-Jaramillo

que las familias que participaron en la Escuela para Familias pertenecen a familias extensa, nuclear, monoparental con jefatura masculina/femenina, ensamblada; las familias en ciclo vital con hijos(as) en etapa de niñez y adolescencia pertenecen a estratos entre 1 al 5 . De acuerdo con lo referido por los miembros de las familias durante los encuentros, éstos provienen de contextos de alto riesgo de trabajo infantil y uso inadecuado del tiempo libre. Es así como la mayoría de las familias se encuentra en un proceso iniciado de restablecimiento de derechos y con medida de protección por el Instituto Colombiano de Bienestar Familiar (ICBF), por lo que se establece la asistencia obligatoria a la Escuela para Familias. Asistieron niños y niñas en el ciclo de primera infancia, con edades entre 2 a 5 años; niños y niñas en edades entre de 6 a 11 años; adolescentes entre 12 y 17 años; también dos padres en situación de discapacidad y otras analfabetas. Algunos de los miembros de las familias se encuentran en su mayoría en etapa del ciclo vital juventud, otros en adultez y su minoría en vejez.

Cabe resaltar que la mayoría de la población que asiste a los encuentros es de sexo femenino y de nivel académico bachiller, pero también se identificaron algunos profesionales.

El Instituto Colombiano de Bienestar Familiar (ICBF), es una entidad del Estado que trabaja por la prevención y la protección integral de la primera infancia, la niñez, la adolescencia y demás miembros de la familia, brindando especial atención a aquellos en condiciones de amenaza, inobservancia o vulneración de sus derechos. Es importante mencionar que las escuelas para familias se encuentran adscritas a las defensorías de protección de los diferentes centros zonales, y se llevan a cabo con familias que están en proceso de restablecimiento de derechos; sin embargo, están bajo las líneas de acción para el trabajo con familia según grupos etarios, planteado por el Instituto Colombiano de Bienestar Familiar (ICBF, 2019).

En cada una de las dependencias (defensorías, atención a primera infancia, supervisión) de los centros zonales, participa en los procesos de atención un equipo psicosocial conformado por: trabajador(a) social, psicólogo(a), nutricionista, y defensor(a) de familia, quienes son los responsables de tomar decisiones y acciones frente a la problemática evidenciada durante la intervención con los(as) usuarios(as) afectados(as). Para el caso de las escuelas, los encargados del programa pueden ser un(a) trabajador(a) social o un(a) psicólogo(a) (ICBF, 2019).

En consecuencia, el Instituto Colombiano de Bienestar Familiar (ICBF, 2010) en las líneas de acción para el trabajo con familias del ICBF, grupos etarios, define las Escuelas para Familias como espacios estratégicos en los que la metodología encuentra un lugar fundamental. Se conciben como procesos formativos con las familias que buscan la 
reflexión, el intercambio de experiencias y la creación de alternativas no violentas a situaciones conflictivas con el fin de mejorar sus relaciones y la convivencia familiar (p. 30).

\section{Objeto y ejes de la sistematización}

La Escuela para Familias se constituyó a partir de las preocupaciones derivadas de la práctica pre profesional al intervenir la población de niños, niñas y adolescentes víctimas de delitos sexuales, maltrato infantil y vulneración de los Derechos Fundamentales.

Durante el proceso de práctica se evidenció que la cobertura en la atención integral de la Escuela para Familias no es suficiente, debido a que la disposición de tiempo no es favorable para contener todas las temáticas.

Es así como se abordaron temáticas orientadas al fortalecimiento de las habilidades parentales educativas, con el propósito de que disminuyeran las problemáticas latentes y manifiestas en la población enunciada anteriormente. En cuanto al rol de los(as) practicantes de Trabajo Social, se construyó la propuesta de intervención y se implementó.

\section{Problemática de la sistematización}

En el Centro Zonal Jamundí se identificó que el proceso de intervención ejecutado a través de la Escuela para Familias primero no tiene continuidad, segundo los temas tratados estuvieron conforme a las problemáticas evidenciadas, pero resultaron insuficientes y, por último, dentro de la intervención no fue posible el abordaje de problemáticas distintas a las planteadas en el plan, aunque requieren atención prioritaria. Por otro lado, se logró reconocer que no existe una intervención transversalizada desde el enfoque diferencial y las actividades de la Escuela para Familias son demasiado generalizadas; por tal razón, no responden a la estructura y el funcionamiento familiar, y al ciclo de vida familiar de la población participante.

Durante el proceso de intervención, resultó pertinente la identificación de las transformaciones que emergieron en los participantes al iniciar con el proceso de la escuela y finalizarlo, aportando la extensión de la experiencia a sus redes de inmersión pues, desde las narrativas de los sujetos (familias, practicante y profesionales), este resulta ser un espacio de orientación que entreteje redes de apoyo para exteriorizar las dificultades que vivencian las familias.

Sobre el objeto descrito, los siguientes son los ejes de sistematización: 


\section{Eje central}

El proceso de intervención familiar a través de la construcción de perspectivas compartidas sobre la formulación, la implementación y los resultados posteriores a la participación de los sujetos involucrados en la Escuela para Familias del ICBF de Centro Zonal Jamundí, Valle, durante el año 2018-2019.

Sub-ejes

1. Los principales logros del proceso de formulación en la Escuela para Familias a través de la experiencia de los sujetos involucrados del ICBF en el Centro Zonal Jamundí, Valle, durante el año 2018-2019.

2. La intervención familiar en el proceso de implementación de la Escuela para Familias a partir de la experiencia de los sujetos involucrados del ICBF en el Centro Zonal Jamundí, Valle, durante el año 2018-2019.

3. Los resultados de la experiencia de los sujetos involucrados que participaron en la Escuela para Familias, como elemento que fortalece la intervención familiar del ICBF en el Centro Zonal Jamundí, Valle, durante el año 2018-2019.

\section{Metodología}

En Trabajo Social como lo sugiere Cifuentes-Gil (2016), sistematizar posibilita reflexionar sobre la práctica para construir conocimiento a través de la conceptualización, la comprensión y la potenciación de la experiencia. Además, permite asignar contenido, claridad, profundidad, relevancia social y proyección política a las intervenciones y los diálogos entre colegas. De igual manera, Barnechea-García y Morgan-Tirado (2010) conciben la sistematización como la reconstrucción y reflexión analítica sobre una experiencia, mediante la cual se interpreta lo sucedido para comprenderlo, ésta permite obtener conocimientos consistentes y sustentados, comunicarlos, confrontar la experiencia con otras y con el conocimiento teórico existente, y así contribuir a una acumulación de conocimientos generados desde y para la práctica (p. 103).

Sistematizar el proceso de intervención familiar mediante la construcción de perspectivas compartidas sobre la formulación, la implementación y los resultados posteriores a la participación de los sujetos involucrados en la Escuela para Familias del Instituto Colombiano de Bienestar Familiar (ICBF) de Centro Zonal Jamundí, es una forma de producción de nuevos conocimientos hacía la transformación social, de modo que se comprenden los procesos que se han estado desarrolland y los aprendizajes de cada sujeto, con el propósito de contribuir al mejoramiento de las prácticas. 
Silva-Minotta, Forero-Izquierdo y Jaramillo-Jaramillo

El tipo de sistematización se comprende como una sistematización retrospectiva y es agenciada por sujetos que hicieron parte de la experiencia. Asimismo, el propósito es reconstruir la experiencia desde los mismos sujetos que participaron sistematizando el proceso de intervención familiar en la Escuela para Familias del Centro Zonal Jamundí. La sistematización está focalizada en la transición del proceso desde tres perspectivas que son: la formulación, la implementación y los resultados posteriores a la experiencia.

La sistematización retrospectiva hace referencia a lo esbozado por Weick (1995) que explica: al ser más reflexivos y críticos respecto de la propia experiencia pasada, existen más posibilidades de lograr una nueva estabilidad y de encontrar un sentido compartido vertiginosamente.

Los enfoques epistemológicos que guían la sistematización son: el histórico-dialéctico y el dialógico e interactivo. El enfoque histórico-dialéctico, como lo sugiere Ghiso (1998), explica y analiza las dimensiones de las prácticas desde el sentido que otorgan los sujetos, el manejo de las relaciones de poder y la reconstrucción de la experiencia.

Ahora bien, el enfoque histórico-dialéctico permite que se promuevan procesos participativos en el que se comprende no sólo la condición individual, sino también de manera colectiva a través del diálogo sobre la experiencia, como es el caso particular de la Escuela para Familia del Instituto Colombiano de Bienestar Familiar (ICBF) de Centro Zonal Jamundí, Valle. Este enfoque inicia con la experiencia desde las prácticas sociales e históricas que trascienden la interpretación crítica de la realidad para leerla y comprenderla de forma dialéctica, pues posibilita encontrar la relación cronológica entre las prácticas actuales con las anteriores, a partir de los significados que construyen los sujetos que hacen parte de la experiencia, en este caso los participantes de la Escuela para Familias.

Por otro lado, el enfoque dialógico e interactivo explica que las experiencias son comprendidas como espacios de interacción, comunicación y relación, que son reconocidas a través del lenguaje oral y en las relaciones sociales que se gestan en estos contextos (Ghiso, 1998). En ese sentido, este enfoque construye el conocimiento a través de los referentes externos e internos, esto significa que se tienen en cuenta las experiencias de los participantes desde un espacio participativo y de reflexión constante, en el que las interacciones toman especial relevancia. 


\section{Modelo Operativo}

\section{Etapa I: Aprestamiento del proyecto de sistematización de experiencias}

En esta primera fase se realizó el aprestamiento del proyecto de sistematización de experiencias con los sujetos que participaron de la experiencia (familias, practicante y profesionales), con el objetivo de indagar la disposición y seleccionar de manera voluntaria a los sujetos que participaron en la reconstrucción de la experiencia. Para esto se construyó una presentación del plan de sistematización a través de llamadas telefónicas.

Instrumentos: Plan de sistematización y Listado de participantes

\section{Etapa II: Recuperación de la experiencia escrita de la información}

En esta etapa se procedió a la recolección de información de fuentes secundarias de la experiencia de las escuelas para familias, consignadas en documentos tales como: actas de reunión, las evaluaciones de actividades, los diarios de campo de cada actividad, las hojas de planeación, los informes de actividades, manual de convivencia familiar y registro fotográfico que se construyó durante el desarrollo de la Escuela para Familias. Para esto se efectuó el desplazamiento al Centro Zonal Jamundí, y se almacenó la información en una memoria USB; posteriormente, se elaboró un informe del proyecto de intervención de apoyo y acompañamiento familiar, Escuela para Familias, en el que se consignaron elementos como: los objetivos, las metodologías utilizadas, población objetivo, formulación, planeación, entre otros. También se hizo un análisis preliminar en el que se identificaron los aciertos y desaciertos del proceso de formulación, implementación y resultados de la intervención familiar que se ha estado desarrollando.

Instrumento: Formato del Informe de la Escuela para Familias

\section{Etapa III: Reconstrucción de Memoria - Recolección y análisis de información}

En esta etapa se diseñaron y aplicaron los instrumentos entrevista y micro relatos, con el objetivo de recuperar la memoria, analizar y reflexionar sobre: el proceso de formulación, la implementación, y los resultados de la experiencia de los sujetos que accedieron voluntariamente a participar del proceso de sistematización de la Escuela para Familias.

Se realizaron las siguientes actividades:

- Diseño de la guía de entrevistas que se aplicaron a: ocho familias, un de Trabajo Social, dos Trabajadores sociales y una Psicóloga. Estas entrevistas fueron registradas en audios vía telefónica, posteriormente transcritas. 
Silva-Minotta, Forero-Izquierdo y Jaramillo-Jaramillo

- Diseño de la guía de micro relatos aplicada a 1 Trabajador social y 3 familias. Estos relatos fueron registrados en audios vía telefónica y posteriormente transcritos.

- Elaboración de la caracterización de los participantes (familias, profesionales y practicante) con el fin de ampliar el contexto de los participantes.

- Diseño de la matriz de triangulación de la información de entrevistas y micro relatos.

Instrumentos: Guía de entrevistas semiestructuradas (practicante, profesionales y familias), Guía de micro relatos (profesional y familias), Formato de Caracterización de los participantes de la experiencia, Matriz de triangulación de la información.

\section{Etapa IV: Presentación y socialización final del documento y los resultados}

Esta etapa se construyó a partir de los resultados y las voces de cada uno de los sujetos. Se registró la información en un documento con las recomendaciones que permitieron la elaboración de un modelo de intervención familiar para el Instituto Colombiano de Bienestar Familiar (ICBF), el cual fue presentado a la coordinadora del Centro Zonal Jamundí a través del correo electrónico, con el propósito de aportar y potenciar el proceso que se ha estado desarrollando en la Escuela para Familias.

\section{Fuentes de información}

Las fuentes de información seleccionadas para la elaboración de la sistematización fueron, en primer lugar, los agentes claves, población involucrada directamente en el proceso de atención de la Escuela para Familias como fuentes primarias. Se recolectó la información y se construyó la memoria del proceso con las familias, la practicante y los(as) profesionales a través de técnicas cualitativas (entrevistas y micro relatos). Contrastando la información secundaria que se obtuvo de la revisión documental proveniente de la verificación de actas, registro (escrito y fotográfico), se construyó un informe en el que se registró el análisis preliminar, identificando los aciertos y los desaciertos del proceso de implementación de la intervención familiar que se ha estado desarrollando.

\section{Hallazgos}

Se describen los resultados en dos perspectivas a través de técnicas como la entrevista y el microrrelato dirigidas a los sujetos involucrados en la participación de la Escuela para Familias. En cuanto a la entrevista, se utilizaron instrumentos de entrevista semiestructurada y se orientaron en tres momentos: un momento inicial que convocó a los sujetos a que narraran sus expectativas sobre el proceso de formulación de metodologías y 
encuentros previos de la Escuela para Familias; un segundo momento, es la implementación, en la que se desarrollaron talleres y se recopilaron las experiencias de los(as) participantes sobre temáticas, sentimientos y aprendizajes; y un último momento, fue el actual o posterior a la experiencia, compilado desde la memoria y la voz de los sujetos.

A continuación, se presenta el análisis de los relatos y sus categorías:

Las entrevistas evidenciaron lo sucedido antes, durante y después del proceso, y la incidencia en la vida familiar y social. Se realizaron doce entrevistas a: ocho familias, dos profesionales de Trabajo Social, una practicante de Trabajo Social y una psicóloga; cada uno de estos sujetos, participaron de los diferentes ciclos de la Escuela para Familias y relataron la experiencia de todo el proceso, la cual fue articulada a los ejes de la sistematización, la teoría y la construcción de las categorías de análisis que se asociaron a las narrativas de los sujetos involucrados en la experiencia.

\subsection{Categoría familias}

\section{Eje 1: Situación inicial}

En primer lugar, se puede distinguir en el proceso de las entrevistas que las familias consideran la Escuela para Familias como guía, orientación y formación que aporta a la enseñanza y los aprendizajes de la vida personal y familiar. Asimismo, en su imaginario responden que es un lugar donde van a instruirlos acerca de algo, porque es lo que se ha construido socialmente $\mathrm{y}$, porque el término escuela generalmente es asociado a la educación. Por otro lado, se debe tener en cuenta que existe un concepto acerca de lo que es el Instituto Colombiano de Bienestar Familiar (ICBF), muchos tienen el pensamiento de que es un lugar donde se les brinda orientación y ayuda a los problemas familiares.

Al respecto, las familias que participaron en la Escuela para Familias expresaron lo siguiente:

La escuela para familias es una guía para orientarnos sobre los cuidados de nuestros hijos y en la convivencia con ellos" (Entrevista semi estructurada, -J. A. D., Jamundí, marzo 31 de 2020). “(...) Para mí es una orientación, volver a retomar el rumbo de lo que debe ser, porque muchas veces nosotros actuamos de la forma que queremos y en realidad pensamos en lo de nosotros, pero no en el bienestar de nuestros hijos (...) (Entrevista semi estructurada, -R. R., Jamundí, abril 1 de 2020).

La escuela para familias como proceso de formación, genera un espacio de reflexión a las prácticas e incita a la transformación de estas. De modo que, ambos actores, familias e institución garanticen el cumplimiento de lo establecido en la Constitución a través de las leyes basadas en los principios de igualdad, derechos y deberes. Por lo que, en la intervención familiar, se deben llevar a cabo acciones educativas que correspondan con las 
necesidades de aprendizaje de los(as) cuidadores(as), padres, madres u otros familiares para aportar al fortalecimiento de las dinámicas familiares y relación con su entorno. No obstante, no se cuenta con una guía específica que determine un plan de ruta y acción de la Escuela para Familias, por lo que se pretende la construcción de este a partir de la experiencia realizada.

En consecuencia con lo anterior, la practicante responsable de la Escuelas para Familias expresó lo siguiente:

\begin{abstract}
Una escuela para familias es una estrategia educativa para orientar a los padres sobre las pautas de crianza que aplican con los hijos, además se atienden casos diversos, por ejemplo, de violencia intrafamiliar o de acuerdo con lo referido por los operadores, ya que esto mejora la calidad de vida, el bienestar de vida y el desarrollo de los menores y de los padres, los núcleos familiares y cualquier tipo de familia (Entrevista semi estructurada, - G. L., Jamundí, abril 1 de 2020).
\end{abstract}

Las propuestas de cada uno de los actores para el desarrollo de las temáticas disponen de la articulación de las necesidades, pero, sobre todo, la colaboración con el tejido de red institucional orientada a la promoción y la prevención de situaciones que la familia puede enfrentar. En Colombia, la Política Pública Nacional de Apoyo y Fortalecimiento a las Familias publicada por el Ministerio de Salud y Protección Social (2016) dispone como objetivo general reconocer, promover y fortalecer las capacidades de las familias como sujetos colectivos de derechos y protagonistas del desarrollo social (p. 18), con el propósito de apoyar y fortalecer el desarrollo social de la población desde su diversidad, como protagonista de su propio cambio a través del empoderamiento de sus prácticas y avance en el cumplimiento de sus compromisos.

\title{
Eje 2: Implementación de la experiencia
}

Considerando la coparticipación de las familias, se crearon escenarios para que observen sus interacciones y las de otros, para aprender de estas, generando cambios e interpretaciones en la forma en que se relacionan con los miembros al interior y al exterior. Por un lado, algunas manifestaron su interés con respecto a que la Escuela presente una continuidad, pero a su vez que la participación de los miembros de la familia sea de forma integral por cada sujeto que la compone.

Conforme a lo previo, un miembro de la familia que participó en la Escuela para Familias expresó lo siguiente:

No, lo único a decir, pues que siga. Lo de la escuela es bueno porque hay personas, vuelvo y le digo, que a veces piensa uno que está actuando bien ante sus hijos, a veces no es así, se da uno cuenta que no es así en varias cosas, en la forma de hablarles, de reprenderlos, de la atención. Entonces es bueno para que la escuela siga y de pronto alguien que pase por lo mismo que yo, 
que sepa que está corrigiéndolos bien, que la escuela siga, eso ayuda mucho. (Entrevista semi estructurada, -M. Q., Jamundí, 2020).

En este caso se puede afirmar, a partir de Schnitman (2008), que el trabajo con familias aborda diálogos reflexivos para concertar y diseñar soluciones, que permita transformar las perspectivas y pautas de relaciones familiares y sociales, ajustando y recreando estrategias de intervención y acción, a partir de otros futuros posibles, actuando sobre las circunstancias presentes. Pues imaginar "una realidad alterna" es al mismo tiempo imaginar las acciones que conducen a ésta y, por lo tanto, otra existencia para quien la imagina; y a su vez utilizar sus propias reflexiones para mejorar la comprensión.

Con relación a lo anterior, una de las profesionales responsables de la Escuela para Familias expresó lo siguiente:

(...) de mi intervención particular el objetivo específico estaba centrado, por un lado, en fortalecer el vínculo afectivo entre padres e hijos y, por otro, en favorecer el conocimiento y construcción de prácticas efectivas de crianza que fuesen alternativas a las que se conocen tradicionalmente; con respecto al primer objetivo, se habló también de las diferentes etapas del desarrollo y como el vínculo se transforma en las diferentes etapas, así como de qué manera es recibido de formas diferentes por los hijos, y no todos tienen un mismo estilo de comunicación o de vínculo afectivo con los padres (...). (Entrevista semiestructurada, -E. U., Jamundí, 01 abril de 2020)

Recuperar los significados derivados de su experiencia pasada y generar conocimiento a partir de la misma facilita nuevos aprendizajes compartidos resultantes del quehacer para la innovación a partir de las prácticas cotidianas.

\section{Eje 3: Resultados posteriores a la experiencia}

Uno de los logros que puede visibilizarse a nivel personal en cada familia es cómo, a través de los procesos dialógicos de compartir las experiencias, las familias aprenden de los(as) otros(as) porque el proceso de unos(as) refleja aspectos de los(as) otros(as) del cual pueden aplicar herramientas que funcionen para el mejoramiento de su dinámica familiar.

Es así como las interacciones que se gestan entre la familia y su entorno afectan a sus miembros y a su vez al vínculo familiar, ya que el tipo de relaciones establecidas incide de forma significativa en cómo la familia hace frente a las problemáticas.

En los relatos de los participantes, se logra identificar un factor común y es la importancia de los vínculos familiares, el fortalecimiento de estos, de acuerdo con la estructura y composición familiar. Además, es importante resaltar que el profesional conozca las relaciones establecidas entre los miembros de la familia, ya que estas condicionan la intervención familiar. 
En contraste, en los relatos de las familias se puede evidenciar que después de la intervención de la Escuela para Familias se observó un cambio en los patrones de comportamiento de los miembros, pero también en las relaciones que se gestan dentro de la estructura familiar, donde cada uno de sus miembros, en este caso la madre y el padre, pueden estar en un ciclo de vida y traer patrones distintos, y además tener un proceso de socialización que influya en cómo se relacionan con sus hijos(as) en la actualidad.

Resulta que en los discursos de las tres familias se pueden evidenciar dos elementos comunes: el primero es la importancia de tener en cuenta a los sujetos de intervención, sus necesidades, sus intereses, los temas en los que necesitan apoyo y fortalecimiento para la transformación e implementación de los procesos, pues en la mayoría de las ocasiones de eso depende el éxito de los mismos; y el segundo es la capacidad del profesional y la institución de adaptarse a todas las situaciones que se presentan y buscar la forma de dar continuidad a los procesos.

Al respecto, las familias participantes en la Escuela para Familias expresaron lo siguiente:

(...) las fortalezas fue el aprendizaje, el conocimiento de muchos temas que no tenía. Bueno, sí tenía el conocimiento, pero no sabía cómo darle el manejo" (Entrevista semiestructurada - D. C., Jamundí- 01 abril de 2020). “(...) Aprendí que los niños también tienen derecho a expresarse, lo que siente en su momento y como padres escucharlos y el tema de la convivencia, de sacarle tiempo a los niños, así no sea mucho tiempo, que sea tiempo de calidad para que vayan siendo unos seres buenos a futuro (...). (Entrevista semiestructurada - R. R., Jamundí- 01 abril de 2020).

Adicional a esto, Schnitman (2008) propone que el trabajo con familias se aborde a partir de diálogos reflexivos para concertar y diseñar soluciones, posibilitando transformar las perspectivas y pautas de relaciones familiares y sociales, ajustando y recreando estrategias de intervención y acción. Con base en lo previo, se lo logra evidenciar en las narrativas de las familias el proceso de intervención fundamentado en las experiencias, los diálogos, se propició la reflexión y se construyó conocimiento a partir de esos elementos.

\subsection{Categoría por familias y practicantes}

\section{Eje 1: Situación inicial}

La forma en la que se concibió la Escuela para Familias desde un sujeto que hace parte de la formulación del proceso, es que las estrategias que este espacio proporcionó mejoran el bienestar social y el desarrollo de la familiar. Por otra parte, al indagar la opinión de las familias sobre la Escuela para Familias se destacaron aspectos metodológicos que facilitan la educación de sus hijos(as) con responsabilidad y valores, que les permiten tener una 
adecuada formación de la personalidad, como elemento protector contra cualquier tipo de violencia o vulneración de derechos.

En correspondencia con lo previo, un profesional responsable de la Escuela para Familias expresó lo siguiente:

(...) me ayudó a fortalecer mucho, a nivel personal la paciencia, aprender a escuchar, ser un buen receptor para ser un buen emisor, aprender a escuchar del núcleo familiar cuando una persona tiene la razón, aprender a escuchar y entender a las personas. (Entrevista semiestructurada - J. M. L., Jamundí, 01 abril de 2020).

En cuanto al desarrollo de las actividades planteadas, la practicante menciona la superación de los NNA de los(as) cuidadores(as), padres, madres u otros miembros de las familias como estrategia, contrario a lo que las familias comunican ante la necesidad de compartir los espacios para que de manera conjunta se elaboren estrategias que permitan ver las falencias tanto a cuidadores(as), padres, madres u otros miembros de la familia.

Además, se manifiesta de manera continua por ambos participantes, en mayor medida las familias, que la labor institucional debe estar orientada a las familias, anticipando las situaciones de vulneración de derechos.

Eje 2: Implementación de la experiencia

La practicante mencionó algunas experiencias exteriorizadas por las familias al asistir a la escuela, explicando que la Escuela para Familias fue un proceso que posibilitó el mejoramiento de la dinámica familiar y el desarrollo de las actividades efectuadas. Por lo tanto, se discutió si los contenidos abordados en la Escuela para Familias tienen en cuenta las interacciones a partir de la etapa en la que se ubica la familia. Según lo que expresó la practicante, se abordaron temas con mayor relación a la etapa de la adolescencia, quizás dejando por fuera las demás etapas que podrían tener importancia en la prevención y la promoción de situaciones que pueden estar vivenciando las familias.

Además, es importante disponer de un diagnóstico inicial en el proceso y contrastarlo con los resultados finales. Sin embargo, en la revisión documental se encontró únicamente la información del formato, pero no la aplicación de éstos y los resultados.

Para el caso de la Escuela para Familias algunos sujetos refirieron que la mayoría de las familias que hacen parte estos espacios son de tipología extensas, reconstruidas y monoparentales. Hay que reconocer la necesidad de plantear una propuesta que sirva de referente para llevar a cabo el proceso de la Escuela para Familias, de tal manera que genere continuidad y llegue al alcance de varias familias con procesos de restablecimiento de 
derechos; adicionalmente, en los escenarios como las juntas de acción comunal para fortalecer el desarrollo familiar.

\section{Eje 3: Resultados posteriores a la experiencia}

Teniendo en cuenta lo que expresan los participantes acerca de la experiencia, se pueden resaltar tres aspectos, el primero es la especificidad de la disciplina de los profesionales que intervinieron como son: la psicología, quien como se expresa en el relato, interviene al sujeto desde su individualidad y el mundo interno; y el Trabajo Social, que interviene desde el valor comunitario de las familias, el fortalecimiento de los valores entre otros. Por otro lado, y como segundo postulado, se debe reconocer la importancia del trabajo interdisciplinario ya que es fundamental, la relación de Trabajo Social con otras profesiones que aportan sus conocimientos alcanzados, para que desde allí se renueven aspectos conceptuales, metodológicos y técnicos (Cifuentes-Gil y Camelo, 2005), para el mejoramiento constante de las intervenciones, en este caso de familia. Tercero y último, propender por el trabajo en red, sin dejar de lado, las experiencias de los participantes y los aportes que estas pueden contribuir a la intervención, es aquí donde Trabajo Social cobra gran relevancia porque adquiere varios roles tales como compañía social, apoyo emocional, guía cognitiva y consejos, regulación social, ayuda material y de servicios, y acceso a nuevos contactos.

En ese orden de ideas, se encuentra que existen elementos comunes en las narrativas de los entrevistados, como por ejemplo que, no solo se deben tener aptitudes, sino actitudes acordes con la población, esto quiere decir que más allá de disponer de todos los conocimientos también se debe atesorar la capacidad para ponerse al nivel de las familias, en el sentido de darles protagonismo a ellos y sus experiencias para desde ahí construir juntos, sin perder las competencias de orientador y guía del proceso.

Finalmente, mediante la narrativa de unas experiencias particulares, los profesionales tuvieron la posibilidad de adquirir un conocimiento que de una u otra forma enriqueció sus vidas.

\subsection{Categoría por familias y profesionales}

\section{Eje 1: Situación inicial}

La Escuela para Familias es un espacio de formación que permite compartir las inquietudes del proceso de crianza de los(as) hijos(as), a su vez, las familias exponen la necesidad de que estos espacios no sólo se brinden cuando hacen parte del proceso de 
restablecimiento de derechos, sino, más bien, se promuevan para la prevención de diversas situaciones. Lo anterior, referido por los profesionales.

Asimismo, el profesional concibe que las necesidades de las familias -como sujetos de intervención- son el pilar en el desarrollo de su quehacer aunque, explorando retrospectivamente la experiencia, se interpreta que no logran apuntar de manera directa a los objetivos como lo destacan algunas familias.

\section{Eje 2: Implementación de la experiencia}

Se plantea que el objetivo de la Escuela para Familias es, por un lado, el fortalecimiento del vínculo familiar y, por otro, el brindar herramientas y favorecer el conocimiento para la construcción de aprendizajes significativos. Es así como el ICBF en su lineamiento técnico de inclusión a las familias, aprobado mediante la Resolución $\mathrm{N}^{\circ}$ 002366, donde se expiden las rutas de atención a las familias y comunidades, teniendo en cuenta aspectos como los territorios, el enfoque diferencial, las comunidades étnicas, entre otras (en cumplimiento de la Política Pública de apoyo y fortalecimiento a las familias y la Ley 1361 de 2009), esboza la importancia de tener en cuenta los contextos donde se relacionan las familias para comprender las formas en que se relacionan. Sin embargo, en las narrativas no se evidencia que se realizaron diagnósticos acerca de las familias para conocer su contexto.

Para cumplir el objetivo, el ICBF propone un aprendizaje generativo, que consiste en aprender de forma colectiva, en este sentido se le debe dar valor a la recuperación de la experiencia pasada y generar conocimiento a partir de la actual, esto con el fin de facilitar la elaboración de aprendizajes significativos, además de promover formas distintas de intervención, pues la participación a través del diálogo es un elemento fundamental del proceso, dado que las familias al interactuar con otras, aprenden de ellas y generan cambios e interpretaciones de sus mismas dinámicas. Por lo anterior, es de vital importancia propiciar escenarios dialógicos y lúdicos para la adecuada interacción e interpretación de experiencias.

Desde las palabras de las familias, se logra evidenciar que la Escuela para familias representó un proceso de aprendizajes lúdicos y participativos, que requiere de un compromiso colectivo, de la institución, los profesionales y las familias. Para finalizar, las habilidades que destacaron las familias de los profesionales son: la claridad de los temas, la practicidad de la enseñanza y la metodología didáctica. 


\section{Eje 3: Resultados posteriores a la experiencia}

Se puede entender desde el discurso de las familias que el proceso de la Escuela para Familias aparentemente cumplió con los objetivos planteados, pues se generó un proceso de reflexión y diálogo de saberes entre los participantes. Dijeron que efectivamente mejoraron muchos aspectos de la vida familiar, tales como la convivencia y la comunicación entre los miembros, además de la importancia del tiempo en familia y la comprensión; en otras palabras, se generó un proceso de introspección familiar y de revisión de todas las dinámicas en su interior.

Por otra parte, se evidenció la capacidad de introspección de las familias para evaluar las formas de relacionarse con sus hijos(as), las actitudes, los modelos y las pautas de crianza que ejercen en el sistema familiar.

Al respecto, un miembro de una familia participante en la Escuela para Familias expresó lo siguiente:

(...) hay algo que aún estoy trabajándole y es lo límites, la experiencia de los límites era algo que estaba marcando y pienso que, deteriorando la crianza con el niño. Sí, cuando ustedes enseñaban que la firmeza de una forma que necesitamos comprensión, de entender que era el adolescente y cómo irlo abordando, para mí era fortalecer entender y aun continúo pues como trabajándola, aunque yo sé que, con otras ayuditas, me va bien. Entonces lo límites marcaron, como que fue ahí, me dio la clave y era el punto que yo necesitaba en ese momento (...). (Entrevista semi estructurada, -L. J. R., Jamundí, abril 1 de 2020).

Cabe mencionar que, un aspecto en el que los(as) cuidadores(as), padres $\mathrm{u}$ otros miembros de las familias y los(as) profesionales coincidieron es que la Escuela para Familias se constituye como una red de apoyo. Primero institucional, ya que se realiza un trabajo articulado entre instituciones. Para este caso, Bienestar Familiar, Comisaria de Familia y familias y Secretaría de Salud, que destinan los recursos técnicos y humanos para la realización de las actividades. También identificaron el apoyo parcial de la institución, y que el proceso no presenta continuidad y no se hace seguimiento, aunque se rescató el trabajo interdisciplinario que desarrollan los profesionales.

Para finalizar, el seguimiento a las familias se realiza cuando tienen un caso adjudicado de restablecimiento de derechos. Por lo tanto, se debe garantizar que los procesos no se queden solo en los talleres de la Escuela para Familias o en el seguimiento posterior a la experiencia desde el reduccionismo de los casos. 


\subsection{Categoría por practicantes y profesionales}

\section{Eje 1: Situacional inicial}

Los profesionales y la practicante relacionan el objetivo principal de la Escuela para Familias de brindar herramientas a los(as) cuidadores(as), padres, madres u otros miembros de la familia con el propósito de abordar las problemáticas que surgen alrededor del proceso de crianza de sus hijo/as, de acuerdo con el ciclo de vida en el que la familia se encuentre. Esto hace referencia a que la Escuela para Familias se cuestione el desarrollo de las sesiones educativas, articulados a la realidad contextual de las familias que se incorporan en el proceso.

Con respecto a lo anterior, un profesional responsable de la Escuela para Familias expresó lo siguiente:

\footnotetext{
(...) en todos los talleres el objetivo era garantizar herramientas a los padres, proporcionarlas. Sabemos que en convivencia familiar es un tema, pues, más complejo de aprender y de aplicar, yo siempre tuve la intención de hacerlo muy vivencial y experiencial, a modo de práctica en el taller y a modo de tarea para la casa (...). (Entrevista semiestructurada, -J. O., Jamundí, abril 01 de 2020).
}

Cierto es que la intervención psicosocial con cuidadores(as), padres, madres u otros miembros de la familia está orientada hacia la creación de herramientas de reflexión, de conversación y de prácticas que generen aprendizajes significativos que trasciendan las dinámicas individuales, familiares y sociales. Otro aspecto que se consideró es que se extienda la convocatoria en la Escuela para Familias, con el fin de que asistan de manera voluntaria otras familias que no estén vinculadas a procesos de restablecimiento de derechos.

Por otro lado, se evidencia en el discurso de los profesionales que no se tienen en cuenta las necesidades de la población al momento de plantear la metodología y los temas de la escuela, ya que en las primeras intervenciones es donde se identifican esas necesidades, esto quiere decir que no se realiza un diagnóstico adecuado para formular el plan de acción.

\section{Eje 2: Implementación de la experiencia}

En este eje tanto profesionales como la practicante coinciden en que las temáticas abordadas en la Escuela para Familias son reduccionistas porque se focalizan en los siguientes temas como: las pautas de crianza, la comunicación, los tipos de violencia y el consumo de sustancias psicoactivas. También, mencionaron que no existe un énfasis en la transversalización de la intervención desde los enfoques de género e intercultural, ya que se limita solamente al enfoque de derechos desde su generalidad. Además, no se tiene en 
cuenta la etapa del ciclo vital de las familias, puesto que son abordadas de manera estandarizada.

En cuanto al objetivo de la Escuela para Familias, se evidencia que es reconocido por todos los sujetos que se incorporaron a la experiencia, ya que concuerdan que está orientado al fortalecimiento de los vínculos y al desarrollo familiar. Por último, se deben tener en cuenta las condiciones socioeconómicas de las familias, pues según las narrativas de la practicante y trabajadores sociales, son familias de estrato socioeconómico bajo que trabajan en su mayoría en el sector obrero, también el reconocimiento de su tipología familiar.

Conforme a lo anterior, una profesional responsable de la Escuela para Familias expresó lo siguiente:

\begin{abstract}
Recuerdo que la mayoría eran padres o madres de familia pertenecientes a estrato socioeconómico medio bajo, más inclinándose hacia un estrato bajo, la mayoría de las personas están desempeñándose dentro del sector de servicios, del sector obrero, algunos creo que eran independientes, aunque no los conocí a profundidad. Un aspecto que compartían en común era que todos se encontraban gestionando procesos dentro del ICBF relacionados con la relación y el vínculo que tenían con sus hijos. (Entrevista semiestructurada, -E. U., Jamundí, abril 1 de 2020).
\end{abstract}

\section{Eje 3: Resultados posteriores a la experiencia}

Se logra evidenciar desde el relato de los profesionales y la practicante que coexiste la representación de la experiencia desde expresiones que la enaltecen. Por otra parte, las familias destacaron las competencias de los profesionales y la practicante caracterizadas en el liderazgo, el manejo de grupo, la capacidad de tener empatía, la buena disposición, un lenguaje adecuado y no muy técnico, además del dominio de los temas a tratar y la capacidad para comprender las dinámicas que se gestan dentro del grupo.

Por otro lado, más allá de poseer aptitudes, se deben tener actitudes acordes con la población, esto significa que se procura por desarrollar la capacidad para ponerse al nivel de las familias, en el sentido de darles protagonismo para que construyan sus experiencias, sin perder la capacidad de ser orientadores del proceso.

Al respecto, un profesional responsable de la Escuela para Familias expresó lo siguiente:

Un muy buen manejo de grupo, es decir, muy buena receptividad, una buena capacidad de identificar las sensaciones en el ambiente o en el transcurso del taller para sí mismo ir direccionando y manejando esos cambios de ritmo durante el mismo, porque si lo hace bien, va a poder transmitir de manera muy sencilla el propósito del taller o lo que se quiere lograr (...). (Entrevista semiestructurada - J. O., Jamundí- abril de 2020). 
Silva-Minotta, Forero-Izquierdo y Jaramillo-Jaramillo

En cuanto a lo institucional, el Instituto Colombiano de Bienestar Familiar como ente encargado de la protección de la niñez, la adolescencia y las familias, y en cumplimiento de la Ley de protección integral a la familia y la política pública de apoyo y fortalecimiento a las familias, debe garantizar todos los recursos físicos, económicos, humanos e institucionales para los programas de desarrollo familiar; sin embargo, se evidenció en las narrativas de la practicante y los profesionales que no hubo un compromiso real por parte de las instituciones involucradas en el proceso de Escuela para Familias. Esto se demostró en la poca disposición de contratación de los profesionales y además de que manifestaron que el ICBF invisibiliza la Escuela para Familias como un Programa fundamental para el desarrollo familiar y social, ya que no dispone de recursos suficientes que permiten la continuidad y la oportunidad del seguimiento de la población.

Para terminar, la formulación de las estrategias para fortalecer escenarios educativos, dialógicos y lúdicos se debe articular en corresponsabilidad institucional y responsabilidad de los(as) cuidadores(as), padres, madres u otros miembros de la familia para asistir a las sesiones que ofrece el Instituto Colombiano de Bienestar Familiar (ICBF) en su estrategia de Escuela para Familias.

\section{Conclusiones}

A partir del análisis realizado y la información recolectada durante el proceso de sistematización, se concluyó lo siguiente:

1. Es fundamental, generar procesos de intervención con familias de manera participativa que conduzcan a reflexiones en los participantes y se construya el conocimiento desde las experiencias y los saberes compartidos, que están articulados a las teorías y el objetivo esencial de la Escuela.

2. El Instituto Colombiano de Bienestar Familiar (ICBF) dispone de guías y líneas de acción que son primordiales para la intervención familiar, ya que contienen metodologías, temáticas y supuestos teóricos que posibilitan una mejor comprensión de la realidad. Lo anterior debería ser socializado con los participantes de la Escuela para Familias, quienes manifestaron que desconocen esta información.

3. El trabajo en red es importante, esto se evidenció en la Escuela para Familias que trabajó de manera articulada con la Secretaría de Salud Departamental, la Comisaría de Familia y los centros zonales del Instituto Colombiano de Bienestar Familiar (ICBF), generando procesos de corresponsabilidad institucional e interviniendo de forma transdisciplinar para lograr como resultado mayor participación de la población objetivo. 
Silva-Minotta, Forero-Izquierdo y Jaramillo-Jaramillo

4. Las Escuelas para Familias no disponen de un proceso de seguimiento posterior a los encuentros, por tal razón, no ha sido posible el reconocimiento de los procesos de transformación de las dinámicas familiares.

5. Teniendo en cuenta las narrativas de los sujetos (familias, practicante y profesionales), con relación a la percepción de la Escuela para Familias, coincidieron en simbolizarla como un espacio de orientación que entreteje redes de apoyo para exteriorizar las dificultades que vivencian las familias en las etapas del ciclo vital. Así mismo, son espacios de promoción de bienestar y prevención del riesgo psicosocial.

6. El enfoque sistémico plantea que la familia es un conjunto interdependiente y organizado de personas que se relacionan e interactúan entre sí, y también con su entorno; no obstante, las familias no son estáticas, se encuentran en constante cambio y transformación, por lo cual es importante que la intervención familiar se sustente en elementos teóricos, que correspondan a las necesidades y objetivos que se quieren alcanzar, además de realizar las modificaciones correspondientes de acuerdo a las exigencias del proceso. En este caso, fue importante tener en cuenta el ciclo vital de la familia, a los sujetos y sus necesidades, y los lineamientos que plantea el Instituto Colombiano de Bienestar Familiar (ICBF) con respecto al desarrollo familiar.

\section{Referencias bibliográficas}

Barnechea-García, M., y Morgan-Tirado, M. (2010). La sistematización de experiencias: producción de conocimientos desde y para la práctica. Tendencias \& Retos, (15), 97-107. Recuperado de http://www.ts.ucr.ac.cr/binarios/tendencias/rev-co-tendencias-1507.pdf.

Cifuentes-Gil, R. M. (2016). Sistematización de Experiencias en Trabajo Social: Desafío Inminente e Inaplazable. Recuperado de https:/ / cepalforja.org/sistem/bvirtual/wpcontent/uploads/2016/10/Sistematizaci\%C3\%B3n-de-exps-en-TS-desaf\%C3\%ADoInminente-e-inaplazable-RMCG-dic-010.pdf.

Cifuentes-Gil, R. M., y Camelo, A. (2005). Aproximaciones a la comprensión del Trabajo Social: línea de construcción disciplinar Facultad de Trabajo Social de la Universidad de La Salle. Tendencias $\mathcal{E}$ Retos, (10), 65-85.

Ghiso, A. (1998). De la práctica singular al diálogo con lo plural. Aproximaciones a otros tránsitos $y$ sentidos de la sistematización en épocas de globalización. Recuperado de https:/ / cepalforja.org/sistem/bvirtual/ $? \mathrm{p}=661$.

Instituto Colombiano de Bienestar Familiar [ICBF]. (2010). Líneas de Acción para el trabajo con familias en el ICBF por grupos etarios. Bogotá, Colombia: ICBF

Instituto Colombiano de Bienestar Familiar [ICBF]. (2019). El instituto. Recuperado de https://icbf.gov.co/instituto. 
Silva-Minotta, Forero-Izquierdo y Jaramillo-Jaramillo

Ministerio de Salud y Protección Social. (2016). Política Pública de apoyo y fortalecimiento a las familias. Bogotá, Colombia: Minsalud. Recuperado de https:/ /www.icbf.gov.co/sites/default/files/politica_de_apoyo_y_fortalecimiento_ a_las_familias-marzo2018.pdf.

Schnitman, D. F. (2008). Diálogos generativos. En G. Rodríguez-Fernández (Coord.), Diálogos Apreciativos: el socioconstruccionismo en acción (pp. 17-48). España: Dykinson.

Torres-Carrillo, A. (2010). Educación Popular y producción de conocimiento. La Piragua, (32),

$8-25$.

Recuperado

de https:/ / ceaal.org/images/stories/LaPiragua/la\%20piragua\%2032\%20v.pdf.

Weick, K. E. (1995). Sensemaking in Organizations. Thousand Oaks, Estados Unidos: SAGE. 


\section{OTROS ARTÍCULOS DE PROSPECTIVA No. 33}

\section{EDITORIAL}

El Paro Nacional en Colombia 2021: explosión social entre dinámicas estructurales y de coyuntura. Relevancia de la acción política y del diálogo en su desarrollo y transformación Adolfo Adrián Álvarez-Rodríguez

\section{ARTÍCULOS}

Organizaciones sociales de pobladores y prácticas de resistencia en contexto de pandemia COVID-19 en Chile

Patricia Castañeda-Meneses

Entre el aislamiento y las brechas digitales: sistematización de experiencia de acompañamiento socioemocional en personas mayores de Temuco, Chile, en tiempos de COVID-19

G. Bernarda Aedo-Neira

Percepción de apoyo social y calidad de vida: la visión de personas mayores chilenas en el contexto de pandemia durante el 2020

Claudia Elena Quiroga-Sanzana

Gabriela Rocío Parra-Monje

Camila Julia Moyano-Sepúlveda

Marco Alejandro Díaz-Bravo

A propósito del qué, cómo y para qué investigar en el campo de la justicia juvenil: La trastienda de un proceso de investigación en Rosario, Argentina Karina De Bella

Proyección social: reflexión sobre una experiencia virtual con adolescentes en época de pandemia en Sucre y Bolivar, Colombia

Claudia Yaneth Martínez-Mina
La intervención social mediante proyectos comunitarios en modalidad virtual en Mérida, México. Una perspectiva desde el Trabajo Social Claudia Isabel Tzec-Puch Amairani Aracelly Ceh-Alvarado Yanet Guadalupe González-Canul

Resignificación de los vínculos Sociales durante la pandemia de COVID-19: narrativas de personas que convergen en Conjuntos residenciales en Bogotá, Colombia

Andrea Marcela Reyes-García

Laura Daniela Molina-Ortiz

La paradoja de la familia doblemente recluida: clínica de una pandemia en Colombia

Norman Darío Moreno-Carmona

Juan José Cleves-Valencia

Ciencias sociales, mundo y pandemia: por un futuro posible

José Miguel Segura-Gutiérrez

Lina Paola Vásquez-Ávila

¿Qué es Trabajo Social? Los primeros vínculos de los estudiantes con la profesión: experiencias de Argentina, Brasil, Paraguay y Uruguay

Silvia Orieta Rivero-Rodríguez

Leonel Del Prado

Nidia Graciela Battilana-Amarilla

Rosilaine Coradini-Guilherme

Intervención profesional de trabajadores sociales de la universidad del Quindio, Colombia. Asuntos epistemológicos, metodológicos y ético-políticos

Ana María Gil-Ríos 
Reflexiones sobre la intervención social en lo rural: experiencias en el Magdalena Medio, Colombia

Claudia Milena Quijano-Mejía

Johana Linares-García

Construcción de rutas de atención integral a la convivencia escolar en Cali, Colombia: sistematización de la experiencia

Leidy Johana Prado-Montaño

Luz Helena López-Rodríguez

Alejandra Gutiérrez-Cárdenas

Experiencia del proceso de intervención familiar de los actores participantes en la Escuela para Familias del ICBF-Jamundí, Colombia Katherin Viviana Silva-Minotta

María José Forero-Izquierdo

Jimena del Pilar Jaramillo-Jaramillo

\section{RESEÑAS DE LIBROS}

Alerta global: políticas, movimientos sociales y futuros en disputa en tiempos de pandemia Jairo Crispín

El neoliberalismo como teología política. Habermas, Foucault, Dardot, Laval y la historia del capitalismo contemporáneo

José Francisco Desentis-Torres

La Reconceptualización del Trabajo Social en Colombia: Análisis histórico-crítico de las décadas de 1960-1970

Juan Pablo Sierra-Tapiro

ARTISTA INVITADO

Juan Camilo González

Prospectiva

\section{PROSPECTIVA}

Revista de Trabajo Social e Intervención Social

No. 33 • ene.-jun. 2022

e-ISSN: 2389-993X • Universidad del Valle 